\title{
Algebraic construction of current operators in integrable spin chains
}

\author{
Balázs Pozsgay ${ }^{1}$ \\ ${ }^{1}$ MTA-BME Quantum Dynamics and Correlations Research Group, \\ Department of Theoretical Physics, \\ Budapest University of Technology and Economics, \\ 1521 Budapest, Hungary
}

\begin{abstract}
Generalized Hydrodynamics is a recent theory that describes the large scale transport properties of one dimensional integrable models. At the heart of this theory lies an exact quantum-classical correspondence, which states that the flows of the conserved quantities are essentially quasi-classical even in the interacting quantum many body models. We provide the algebraic background to this observation, by embedding the current operators of the integrable spin chains into the canonical framework of Yang-Baxter integrability. Our construction can be applied in a large variety of models including the XXZ spin chains, the Hubbard model, and even in models lacking particle conservation such as the XYZ chain. Regarding the XXZ chain we present a simplified proof of the recent exact results for the current mean values, and explain how their quasi-classical nature emerges from the exact computations.
\end{abstract}

Introduction.- The non-equilibrium dynamics of one dimensional quantum integrable systems has attracted a lot of interest [1]. Integrable models possess a large number of commuting conserved charges, constraining their dynamical processes and leading to dissipationless and factorized scattering. This exotic dynamical behaviour has a number of experimentally measurable consequences, for example a lack of thermalization $[2,3]$. Two central theoretical problems have been the equilibration in isolated integrable models, and the description of transport in spatially inhomogeneous and/or driven systems. Regarding equilibration it is now accepted that the emerging steady states can be described by the Generalized Gibbs Ensemble [4, 5]. Regarding transport the theory of Generalized Hydrodynamics (GHD) was introduced in $[6,7]$, which describes both the ballistic modes and also the diffusive corrections [8-11]. Recent works [12-16] also treated the phenomenon of super-diffusion.

In GHD a central role is played by the current operators describing the flow of conserved quantities. The continuity relations for these flows completely determine the transport at the Euler-scale [6,7]. It is thus of utmost importance to understand the mean currents in local or global equilibria. The works $[6,7]$ argued that in the thermodynamic limit the currents are given by a formula of the type

$$
J=\int d \lambda \rho(\lambda) v_{\mathrm{eff}}(\lambda) h(\lambda)
$$

where $\lambda$ is a rapidity parameter, $h(\lambda)$ is the one-particle charge eigenvalue, $\rho(\lambda)$ is the differential particle density per volume and rapidity, and $v_{\text {eff }}(\lambda)$ is an "effective velocity" that describes the propagation of single particle wave packets in the presence of the other particles [17]. Clearly, this concept is quasi-classical, and it assumes the dissipationless scattering of integrable models.

The formula (1) has received continued attention. It was known that it holds in models equivalent to free bosons or free fermions, where $v_{\text {eff }}(\lambda)=e^{\prime}(\lambda) / p^{\prime}(\lambda)$ is the group velocity [18]. In interacting cases proofs were given in various settings $[6,19-23]$. The paper [24] derived a new and exact finite volume formula for the mean currents in the Heisenberg spin chains, and a connection to long range deformed models was pointed out in [25]. However, the microscopic proofs were not transparent enough and did not fully explain why there exist such simple and exact formulas for the currents. Furthermore, the direct algebraic representation of the current operators was missing.

In this Letter we fill this gap. We make a direct connection to the Quantum Inverse Scattering Method (QISM) pioneered by L. Faddeev and the Leningrad school $[26,27]$. This is the canonical framework to treat local quantum integrable systems. For the first time we show that the QISM also accommodates the current operators, leading to a simplified rigorous derivation of their mean values, corroborating their quasi-classical nature.

Charges and currents. - We consider integrable spin chains in finite volume, given by a local Hamiltonian $\hat{H}$ acting on the Hilbert space $\mathcal{H}=\otimes_{j=1}^{L} V_{j}$ with $V_{j} \simeq \mathbb{C}^{d}$. We assume periodic boundary conditions.

Examples are the XXX, XXZ and XYZ Heisenberg spin chains [26, 28], or the 1D Hubbard model [29]. These integrable models possess a canonical set of local conserved charges $\hat{Q}_{\alpha}$ that are in involution $\left[\hat{Q}_{\alpha}, \hat{Q}_{\beta}\right]=0$, such that $\hat{H}$ belongs to the family. The charges can be written as $\hat{Q}_{\alpha}=\sum_{x} \hat{q}_{\alpha}(x)$, with $\hat{q}_{\alpha}(x)$ being the charge density operators.

The flow of these charges is described by the current operators $\hat{J}_{\alpha}(x)$, defined through the continuity relations

$$
i\left[\hat{H}, \hat{q}_{\alpha}(x)\right]=\hat{J}_{\alpha}(x)-\hat{J}_{\alpha}(x+1) .
$$

Following $[24,30]$ we also introduce the generalized current operators $\hat{J}_{\alpha, \beta}$ that describe the flow of $\hat{Q}_{\alpha}$ under the time evolution generated by $\hat{Q}_{\beta}$. They are defined 
through

$$
i\left[\hat{Q}_{\beta}, \hat{q}_{\alpha}(x)\right]=\hat{J}_{\alpha, \beta}(x)-\hat{J}_{\alpha, \beta}(x+1) .
$$

It is our goal to compute the exact mean values of $\hat{J}_{\alpha, \beta}$ in the eigenstates of the models, and to show that they always take a form analogous to (1).

Transfer matrices. - The standard method to find the commuting set of charges is the QISM [26, 27]. Below we summarize this procedure; for more details see [26], and for a pictorial interpretation of the main algebraic objects see [31].

We start with the so-called $R$-matrix $R(\mu, \nu) \in$ $\operatorname{End}\left(\mathbb{C}^{d} \otimes \mathbb{C}^{d}\right)$ which satisfies the Yang-Baxter relation:

$$
\begin{aligned}
& R_{12}\left(\lambda_{1}, \lambda_{2}\right) R_{13}\left(\lambda_{1}, \lambda_{3}\right) R_{23}\left(\lambda_{2}, \lambda_{3}\right)= \\
& =R_{23}\left(\lambda_{2}, \lambda_{3}\right) R_{13}\left(\lambda_{1}, \lambda_{3}\right) R_{12}\left(\lambda_{1}, \lambda_{2}\right) .
\end{aligned}
$$

This is a relation for operators acting on the triple tensor product $V_{1} \otimes V_{2} \otimes V_{3}$ and we assume $V_{j} \simeq \mathbb{C}^{d}$. It is understood that each $R_{j k}$ acts only on the corresponding vector spaces. Examples for $R$-matrices (describing the above mentioned models) can be found in [26, 28, 29]. We assume that the so-called regularity and inversion conditions hold:

$$
\begin{aligned}
R(\lambda, \lambda) & =P \\
R_{12}\left(\lambda_{1}, \lambda_{2}\right) R_{21}\left(\lambda_{2}, \lambda_{1}\right) & =1 .
\end{aligned}
$$

Here $P$ is the permutation operator and $R_{21}(u, v)=$ $P R_{12}(u, v) P$.

The charges are obtained from a commuting set of transfer matrices. Let us take an auxiliary space $V_{a} \simeq \mathbb{C}^{d}$ and the Lax-operators $\mathcal{L}_{a, j}(u)$ which act on $V_{a}$ and on a local space $V_{j}$ with $j=1 \ldots L$, where $L$ is the length of the chain. We require that the following exchange relation holds:

$$
R_{b, a}(\nu, \mu) \mathcal{L}_{b, j}(\nu) \mathcal{L}_{a, j}(\mu)=\mathcal{L}_{a, j}(\mu) \mathcal{L}_{b, j}(\nu) R_{b, a}(\nu, \mu)
$$

with $a, b$ referring to two different auxiliary spaces. It follows from (4) that $\mathcal{L}_{a, j}(\mu)=R_{a, j}\left(\mu, \xi_{0}\right)$ is a solution to $(6)$, where $\xi_{0}$ is a fixed parameter of the model. In the following we use this choice and assume that $\xi_{0}=0$.

The monodromy matrix acting on $V_{a} \otimes \mathcal{H}$ is defined as

$$
\hat{T}_{a}(\mu)=\mathcal{L}_{a, L}(\mu) \ldots \mathcal{L}_{a, 1}(\mu) .
$$

The transfer matrix is its partial trace over the auxiliary space: $\hat{t}(\mu)=\operatorname{Tr}_{a} \hat{T}_{a}(\mu)$. The fundamental exchange relations (6) guarantee that $[\hat{t}(\mu), \hat{t}(\nu)]=0$. A generating function for global charges is then defined as [26, 27]

$$
\hat{Q}(\nu) \equiv(-i) \hat{t}^{-1}(\nu) \frac{d}{d \nu} \hat{t}(\nu)
$$

The traditional charges are the Taylor coefficients:

$$
\hat{Q}(\nu)=\sum_{\alpha=2}^{\infty} \frac{\nu^{\alpha-2}}{(\alpha-2) !} \hat{Q}_{\alpha}
$$

The $\hat{Q}_{\alpha}$ are extensive, and the density $\hat{q}_{\alpha}(x)$ spans $\alpha$ sites [32]; in particular $\hat{H} \sim \hat{Q}_{2}$. The definition (8) makes sense in any finite volume, but it gives the correct $\hat{Q}_{\alpha}$ only if $L>\alpha$. In the $L \rightarrow \infty$ limit the operator $\hat{Q}(\mu)$ is expected to be quasi-local in some neighborhood of $\mu=0$, for proofs in concrete cases see [33-35].

Charge densities. - Writing $\hat{Q}(\mu)=\sum_{x=1}^{L} \hat{q}(\mu, x)$ we can identify the corresponding operator density as

$$
\begin{aligned}
\hat{q}(\mu, x) & \equiv(-i) \hat{t}^{-1}(\mu) \times \\
& \times \operatorname{Tr}_{a}\left[\hat{T}_{a}^{[L, x+1]}(\mu) \partial_{\mu} \mathcal{L}_{a, x}(\mu) \hat{T}_{a}^{[x-1,1]}(\mu)\right] .
\end{aligned}
$$

Here we defined the partial monodromy matrices acting on a segment $\left[x_{1} \ldots x_{2}\right]$ as

$$
\hat{T}_{a}^{\left[x_{2}, x_{1}\right]}(\mu)=\mathcal{L}_{a, x_{2}}(\mu) \ldots \mathcal{L}_{a, x_{1}}(\mu) .
$$

The definition (10) is homogeneous in space: $\hat{q}(\mu, x)=$ $\hat{U}^{-1} \hat{q}(\mu, x+1) \hat{U}$, where $\hat{U}$ is the cyclic shift operator to the right.

Eq. (10) is a new result of this work, which serves as a starting point to obtain a similar formula for the currents. It can be considered a Matrix Product Operator (MPO) representation of the charge densities, with a local inhomogeneity at site $x$. For a pictorial representation see [31].

Current operators. - We also construct a generating function for the currents:

$$
\hat{J}(\mu, \nu, x)=\sum_{\alpha=2}^{\infty} \sum_{\beta=2}^{\infty} \frac{\mu^{\alpha-2}}{(\alpha-2) !} \frac{\nu^{\beta-2}}{(\beta-2) !} \hat{J}_{\alpha, \beta}(x) .
$$

This two-parameter family of operators satisfies the generalized continuity relation

$$
i[\hat{Q}(\nu), \hat{q}(\mu, x)]=\hat{J}(\mu, \nu, x)-\hat{J}(\mu, \nu, x+1) .
$$

The summation in (12) only makes sense in the $L \rightarrow \infty$ limit, where we expect that $J(\mu, \nu, x)$ is a finite norm operator localized around $x$, at least in some neighborhood of $\mu=\nu=0$. Relation (13) is well defined in any finite volume, if we use (8)-(10).

It is our goal to give an explicit construction for $\hat{J}(\mu, \nu, x)$. We start with the commutator

$$
\begin{aligned}
& {[\hat{t}(\nu), \hat{q}(\mu, x)]=(-i) \hat{t}^{-1}(\mu) \times} \\
& \quad \times\left.\frac{d}{d \varepsilon} \operatorname{Tr}_{a b}\left(\hat{T}_{b}(\nu) \hat{T}_{a}^{\varepsilon}(\mu)-\hat{T}_{a}^{\varepsilon}(\mu) \hat{T}_{b}(\nu)\right)\right|_{\varepsilon=0},
\end{aligned}
$$

where now $a$ and $b$ refer to two different auxiliary spaces, and $\hat{T}_{a}^{\varepsilon}(\mu)$ is a deformed monodromy matrix defined as

$$
\hat{T}_{a}^{\varepsilon}(\mu)=\hat{T}_{a}^{[L, x+1]}(\mu) \mathcal{L}_{a, x}(\mu+\varepsilon) \hat{T}_{a}^{[x-1,1]}(\mu) .
$$

The modification of the rapidity parameter at site $x$ is the reason for the non-commutativity, and this will result in the appearance of the current operators. 
At $\varepsilon=0$ the intertwining of the monodromy matrices is performed by a repeated application of (6). In $\hat{T}^{\varepsilon}(\mu)$ the difference is that there is one Lax operator with a modified rapidity. At that particular site the exchange is also given by (6), but it involves $R_{b, a}(\nu, \mu+\varepsilon)$. Inserting these commutation relations into (14) and performing the $\varepsilon$-derivative we eventually obtain

$$
\hat{t}^{-1}(\nu)[\hat{t}(\nu), \hat{q}(\mu, x)]=\hat{\Omega}(\mu, \nu, x)-\hat{\Omega}(\mu, \nu, x-1),
$$

where we introduced a new "double row" operator

$$
\begin{aligned}
& \hat{\Omega}(\mu, \nu, x)=\hat{t}^{-1}(\nu) \hat{t}^{-1}(\mu) \operatorname{Tr}_{a b}\left[\hat{T}_{a}^{[L, x+1]}(\mu) \times\right. \\
&\left.\times \hat{T}_{b}^{[L, x+1]}(\nu) \Theta_{a, b}(\mu, \nu) \hat{T}_{a}^{[x, 1]}(\mu) \hat{T}_{b}^{[x, 1]}(\nu)\right] .
\end{aligned}
$$

Here

$$
\Theta_{a, b}(\mu, \nu)=(-i) R_{b, a}(\nu, \mu) \partial_{\mu} R_{a, b}(\mu, \nu)
$$

is an operator insertion acting only on the auxiliary spaces, coupling the two monodromy matrices. A pictorial representation of $\hat{\Omega}(\mu, \nu, x)$ is given in [31].

Taking a further $\nu$-derivative on the l.h.s. of (16) we recognize the continuity equation (13) and identify

$$
\hat{J}(\mu, \nu, x)=-\hat{t}(\nu) \partial_{\nu} \hat{\Omega}(\mu, \nu, x-1) \hat{t}^{-1}(\nu) .
$$

Let $|\Psi\rangle$ be an arbitrary eigenstate of the commuting transfer matrices. For the mean values we get:

$$
\langle\Psi|\hat{J}(\mu, \nu, x)| \Psi\rangle=-\partial_{\nu}\langle\Psi|\hat{\Omega}(\mu, \nu, x-1)| \Psi\rangle .
$$

This connects the $\nu$-derivatives of $\hat{\Omega}(\mu, \nu, x)$ to the current mean values. To complete the picture, we also compute the initial value at $\nu=0$. Direct substitution and the regularity condition lead to $\hat{\Omega}(\mu, 0, x)=\hat{q}(\mu, x)$. Thus $\hat{\Omega}$ not only describes all (generalized) currents, but also all charge densities. Together with (20) this is the first central result of our work.

Symmetry. - We discuss the symmetry of $\hat{\Omega}(\mu, \nu, x)$ under the exchange of its rapidity variables. The partial monodromy matrices in the definition (17) can be exchanged using (6). Direct computation shows that $\hat{\Omega}(\mu, \nu, x)=\hat{\Omega}(\nu, \mu, x)$ iff

$$
\partial_{\mu} R_{b, a}(\nu, \mu)+\partial_{\nu} R_{b, a}(\nu, \mu)=0 .
$$

This is satisfied if the $R$-matrix is of difference form: $R_{b, a}(\nu, \mu)=R_{b, a}(\nu-\mu)$. Examples are the various Heisenberg spin chains, and a famous counter-example is the Hubbard model. This exchange symmetry results in equalities between different charge and current operators, as already observed in [24].

Inhomogeneous cases. - The nature of the operator $\hat{\Omega}$ is better understood if we also consider the inhomogeneous spin chains. Let us take generic complex numbers $\boldsymbol{\xi}_{L}$ and define the inhomogeneous monodromy matrix

$$
\hat{T}_{a}(\mu)=R_{a, L}\left(\mu, \xi_{L}\right) \ldots R_{a, 1}\left(\mu, \xi_{1}\right),
$$

In this case we can still define the $\hat{\Omega}$ operator with formula (17), replacing each local Lax operator with their inhomogeneous versions, and keeping the insertion $\Theta_{a, b}(\mu, \nu)$ the same.

Even though $\hat{\Omega}$ is quite complicated, there is a remarkable simplification when the parameters $\mu, \nu$ are chosen from the set $\boldsymbol{\xi}_{L}$. Let us take for simplicity $\mu=\xi_{1}, \nu=\xi_{2}$ and set $x=2$. A straightforward computations leads to

$$
\hat{\Omega}\left(\xi_{1}, \xi_{2}, 2\right)=\Theta_{1,2}\left(\xi_{1}, \xi_{2}\right) .
$$

This means that for these special values $\hat{\Omega}(\mu, \nu, x)$ becomes an ultra-local operator acting only on the first two sites. This bridges a connection to the theory of factorized correlation functions in the XXZ chain [36-41], where the mean value of $\Theta_{1,2}\left(\xi_{2}, \xi_{1}\right)$ is one of the basic building blocks. Our contribution here is the construction of $\hat{\Omega}(\mu, \nu, x)$ for general $\mu, \nu$, and the explanation that it describes the currents and the charges. The result (23) is also analogous to the "solution of the inverse problem" [42, 43], where the monodromy matrix elements can be specialized such that they become ultra-local operators acting on single sites only.

Mean values. - We return to the homogeneous case and employ a trick originally developed in [39]. We relate the mean values of $\hat{\Omega}(\mu, \nu, x)$ to a transfer matrix eigenvalue in an auxiliary problem. Consider an enlarged spin chain with two extra sites. Choose a rapidity $\mu$ and a deformation parameter $\varepsilon$. The enlarged monodromy matrix acts on $V_{a} \otimes V_{L+2} \otimes V_{L+1} \otimes \mathcal{H}$ and is given by

$$
\hat{T}_{a}^{+}(u)=\mathcal{L}_{L+2}(u) \mathcal{L}_{L+1}(u) T_{a}(u),
$$

where $T_{a}(u)$ is given by (7), and the two extra Lax operators are $\mathcal{L}_{L+2}(u)=R_{a, L+2}(u, \mu+\varepsilon)$ and $\mathcal{L}_{L+1}(u)=$ $R_{L+1, a}^{t_{L+1}}(\mu, u)$, where $t_{L+1}$ denotes partial transposition with respect to the physical space at site $x=L+1$. The Yang-Baxter relation implies that both Lax operators satisfy the exchange relation (6). For $\mathcal{L}_{L+2}(u)$ this follows directly from (6); here $\mu+\varepsilon$ plays the role of an inhomogeneity parameter. For $\mathcal{L}_{L+1}(u)$ it can be proven by taking partial transpose of (6) with respect to the physical space and exchanging the labeling of the rapidities. Putting everything together we can see the transfer matrices defined as $\hat{t}^{+}(u)=\operatorname{Tr}_{a} T_{a}^{+}(u)$ form a commuting set.

At $\varepsilon=0$ the extra two sites become decoupled: If $|\Psi\rangle$ is an eigenstate of the original $\hat{t}(u)$ with eigenvalue $\Lambda(u)$, then

$$
\hat{t}^{+}(u)(|\delta\rangle \otimes|\Psi\rangle)=\Lambda(u)(|\delta\rangle \otimes|\Psi\rangle) .
$$

Here $|\delta\rangle$ is the "delta-state" given by components $\delta_{i j}$ in the computational basis.

After switching on a non-zero $\varepsilon$ the first two sites will affect the eigenvalues and the eigenvectors. Let $\Lambda^{+}(u \mid \mu, \varepsilon)$ be the eigenvalue of $\hat{t}^{+}(u)$ on a state $\left|\Psi^{+}\right\rangle$ 
which in the limit $\varepsilon \rightarrow 0$ becomes $|\delta\rangle \otimes|\Psi\rangle$. A standard first order perturbation theory computation gives [44]

$$
\langle\Psi|\hat{\Omega}(\mu, \nu, x)| \Psi\rangle=\left.i \frac{d}{d \varepsilon} \log \Lambda^{+}(\nu \mid \mu, \varepsilon)\right|_{\varepsilon=0} .
$$

This is the second central result of our work, which applies essentially to "all" Yang-Baxter integrable local chains. The eigenvalues $\Lambda^{+}(\nu \mid \mu, \varepsilon)$ can always be found by standard methods of integrability, and this explains why there exist simple exact formulas for the current mean values. The specifics of the model come into play only when we are actually solving the auxiliary problem.

Heisenberg spin chain.- As an example we take the easy-axis XXZ chain defined by the Hamiltonian density

$$
\hat{h}(j)=\hat{\sigma}_{j}^{x} \hat{\sigma}_{j+1}^{x}+\hat{\sigma}_{j}^{y} \hat{\sigma}_{j+1}^{y}+\Delta\left(\hat{\sigma}_{j}^{z} \hat{\sigma}_{j+1}^{z}-1\right)
$$

Here $\hat{\sigma}_{j}^{x, y, z}$ are Pauli matrices acting on site $j$ and $\Delta=$ $\cosh (\eta)>1$ is the anisotropy parameter. The associated $R$-matrix is of the form

$$
R(\mu, \nu)=\left(\begin{array}{cccc}
1 & 0 & 0 & 0 \\
0 & b(\mu-\nu) & c(\mu-\nu) & 0 \\
0 & c(\mu-\nu) & b(\mu-\nu) & 0 \\
0 & 0 & 0 & 1
\end{array}\right) .
$$

with $b(u)=\sin (u) / \sin (u+i \eta), c(u)=\sin (i \eta) / \sin (u+i \eta)$.

The model can be solved by the Algebraic Bethe Ansatz (ABA) [26]. Eigenstates are labeled by a set of rapidities $\boldsymbol{\lambda}_{N}$, describing $N$ interacting spin waves, satisfying the Bethe equations

$$
p\left(\lambda_{k}\right) L+\sum_{j \neq k}^{N} \delta\left(\lambda_{k}-\lambda_{j}\right)=2 \pi Z_{k}, \quad Z_{k} \in \mathbb{Z},
$$

where $L$ is the length of the chain, and

$$
e^{i p(\lambda)}=\frac{\sin (\lambda-i \eta / 2)}{\sin (\lambda+i \eta / 2)}, \quad e^{i \delta(\lambda)}=\frac{\sin (\lambda+i \eta)}{\sin (\lambda-i \eta)} .
$$

For the generating function of the conserved charges we find the eigenvalues $\hat{Q}(\nu)\left|\boldsymbol{\lambda}_{N}\right\rangle=Q(\nu)\left|\boldsymbol{\lambda}_{N}\right\rangle$ where $Q(\nu) \simeq \sum_{j=1}^{N} h\left(\lambda_{j}-\nu\right)$ and $h(u)=p^{\prime}(u)$. Here and in the following the $\simeq$ sign means that there are correction terms behaving as $\mathcal{O}\left(\nu^{L}\right)$ or $\mathcal{O}\left(\mu^{L}\right)$ for small $\mu, \nu$.

The auxiliary spin chain problem defined by (24) can also be solved using ABA. Here we present the outline of the computation; for the details we refer to [31]. It turns out that the main effect of the extra two sites is that they act as a momentum dependent twist operator for the particles of the original chain. This deforms the Bethe equations and their solutions. We get

$$
-\varepsilon h\left(\lambda_{k}-\mu\right)+p\left(\lambda_{k}\right) L+\sum_{j \neq k}^{N} \delta\left(\lambda_{k}-\lambda_{j}\right) \simeq 2 \pi Z_{k},
$$

where $\mu$ is the external parameter introduced in (24). Furthermore, we have $\partial_{\nu} \log \Lambda^{+}(\nu \mid \mu, \varepsilon) \simeq i Q(\nu)$, where
$Q(\nu)$ is the same function introduced above, but evaluated at the $\varepsilon$-deformed rapidities [31]. Equations (19) and (26) then lead to

$$
\left\langle\boldsymbol{\lambda}_{N}|\hat{J}(\mu, \nu, x)| \boldsymbol{\lambda}_{N}\right\rangle \simeq \sum_{j=1}^{N} h^{\prime}\left(\lambda_{j}-\nu\right) \frac{d \lambda_{j}}{d \varepsilon} .
$$

As a useful trick let us regard the solution $\boldsymbol{\lambda}_{N}$ of (29) as functions of the $Z_{k}$, and let us relax the condition that the $Z_{k}$ are integers. Then the $\varepsilon$-derivatives can be expressed as

$$
\frac{d \lambda_{j}}{d \varepsilon} \simeq \sum_{k=1}^{N} \frac{\partial \lambda_{j}}{\partial\left(2 \pi Z_{k}\right)} h\left(\lambda_{k}-\mu\right) .
$$

Here it is understood that for the physical states the formula is evaluated at integer $Z_{k}$. Then the result (32) is written as

$$
\left\langle\boldsymbol{\lambda}_{N}|\hat{J}(\mu, \nu, x)| \boldsymbol{\lambda}_{N}\right\rangle \simeq \sum_{k=1}^{N} \frac{\partial Q(\nu)}{\partial\left(2 \pi Z_{k}\right)} h\left(\lambda_{k}-\mu\right) .
$$

Expanding to low orders in $\mu$ and $\nu$ we get the final result

$$
\left\langle\boldsymbol{\lambda}_{N}\left|\hat{J}_{\alpha, \beta}(x)\right| \boldsymbol{\lambda}_{N}\right\rangle=\sum_{k=1}^{N} \frac{\partial Q_{\beta}}{\partial\left(2 \pi Z_{k}\right)} h_{\alpha}\left(\lambda_{k}\right) .
$$

Even though the intermediate formulas were only approximate, the final result (35) is exact, and agrees with $[24,25]$; the exact formula for $\left\langle\boldsymbol{\lambda}_{N}|\hat{\Omega}(\mu, \nu, x)| \boldsymbol{\lambda}_{N}\right\rangle$ is presented in [31].

Interpretation.- Consider the semi-classical picture of $N$ particles moving on the circle of circumference $L$, subject to two-particle scattering events described by the phase shift $\delta(\lambda)$ defined above. In this situation $\left(2 \pi Z_{k}\right) / L$ can be interpreted as the "dressed momentum" of the particles, which takes into account the interaction between the particles. Then the formula (35) is interpreted as

$$
\left\langle\boldsymbol{\lambda}_{N}\left|\hat{J}_{\alpha, \beta}(x)\right| \boldsymbol{\lambda}_{N}\right\rangle=\frac{1}{L} \sum_{k=1}^{N} v_{\mathrm{eff}, \beta}\left(\lambda_{k}\right) h_{\alpha}\left(\lambda_{k}\right)
$$

with $v_{\text {eff, }, \beta}\left(\lambda_{k}\right)=L \partial Q_{\beta} / \partial\left(2 \pi Z_{k}\right)$ being the natural generalization of the group velocity under time evolution dictated by $\hat{Q}_{\beta}$. For more details see [24, 25].

Thermodynamic limit. - It is possible to take the thermodynamic limit of (35) with a direct approach, reproducing the results of $[6,7]$. Alternatively, we can apply the Quantum Transfer Matrix approach [39, 41] directly in the thermodynamic limit. These computations will be presented elsewhere.

Discussion.-We constructed a generating function for the charge densities and the current operators using standard tools of Yang-Baxter integrability. The main formulas are model independent. 
Our construction explains why there exist simple formulas for the current mean values: because they are tied to certain transfer matrix eigenvalues through (20) and (26). In integrable models such eigenvalues are always "easy" to compute, in contrast with generic correlation functions, which are much more difficult to handle. This means that the current operators are the "next simplest" operators after the charge densities.

We demonstrated on the example of the XXZ chain that the current mean values have a quasi-classical interpretation. Our derivations suggest that this is a generic feature of integrable spin chains. The ultimate physical reason for this behaviour is the dissipationless and factorized scattering in integrable models, and our work provided new algebraic tools to treat this phenomenon. We stress that our computations are completely rigorous. The approximations above were only introduced to provide a more intuitive understanding. Thus we made an important step towards proving the emergence of hydrodynamics in a quantum many body situation.

In future work we plan to compute the currents in models not yet considered in the literature. A particularly interesting case is the XYZ model, which belongs to the class of models treated here, but lacks particle conservation on the microscopic level. Furthermore, it would be interesting to consider current operators also in the Separation of Variables approach [45-48].

Acknowledgments. - The author is grateful to Frank Göhmann, Benjamin Doyon, Yunfeng Jiang, Márton Kormos, Gábor Takács and Tomaž Prosen for useful discussions and important suggestions about the manuscript. Furthermore we thank Levente Pristyák for checking some of the formulas in the Supplemental Materials. This research was supported by the BMENanotechnology FIKP grant (BME FIKP-NAT), by the National Research Development and Innovation Office (NKFIH) (K-2016 grant no. 119204), by the János Bolyai Research Scholarship of the Hungarian Academy of Sciences, and by the ÚNKP-19-4 New National Excellence Program of the Ministry for Innovation and Technology.

[1] P. Calabrese, F. H. L. Essler, and G. Mussardo, J. Stat. Mech. 2016, 064001 (2016).

[2] T. Kinoshita, T. Wenger, and D. S. Weiss, Nature 440, 900 (2006).

[3] T. Langen, S. Erne, R. Geiger, B. Rauer, T. Schweigler, M. Kuhnert, W. Rohringer, I. E. Mazets, T. Gasenzer, and J. Schmiedmayer, Science 348, 207 (2015), arXiv:1411.7185 [cond-mat.quant-gas].

[4] E. Ilievski, J. De Nardis, B. Wouters, J.-S. Caux, F. H. L. Essler, and T. Prosen, Phys. Rev. Lett. 115, 157201 (2015), arXiv:1507.02993 [quant-ph].

[5] L. Vidmar and M. Rigol, J. Stat. Mech. 6, 064007 (2016), arXiv:1604.03990 [cond-mat.stat-mech].

[6] O. A. Castro-Alvaredo, B. Doyon, and T. Yoshimura,
Phys. Rev. X 6, 041065 (2016), arXiv:1605.07331 [condmat.stat-mech].

[7] B. Bertini, M. Collura, J. De Nardis, and M. Fagotti, Phys. Rev. Lett. 117, 207201 (2016), arXiv:1605.09790 [cond-mat.stat-mech].

[8] J. De Nardis, D. Bernard, and B. Doyon, Phys. Rev. Lett. 121, 160603 (2018), 1807.02414 [cond-mat.statmech].

[9] S. Gopalakrishnan, D. A. Huse, V. Khemani, and R. Vasseur, Phys. Rev. B 98, 220303 (2018), arXiv:1809.02126 [cond-mat.stat-mech].

[10] S. Gopalakrishnan and R. Vasseur, Phys. Rev. Lett. 122, 127202 (2019), arXiv:1812.02701 [cond-mat.stat-mech].

[11] J. D. Nardis, D. Bernard, and B. Doyon, SciPost Phys. 6, 49 (2019), arXiv:1812.00767 [cond-mat.stat-mech].

[12] E. Ilievski, J. De Nardis, M. Medenjak, and T. Prosen, Phys. Rev. Lett. 121, 230602 (2018), arXiv:1806.03288 [cond-mat.stat-mech].

[13] J. De Nardis, M. Medenjak, C. Karrasch, and E. Ilievski, Phys. Rev. Lett. 123, 186601 (2019), arXiv:1903.07598 [cond-mat.stat-mech].

[14] U. Agrawal, S. Gopalakrishnan, R. Vasseur, and B. Ware, arXiv e-prints (2019), arXiv:1909.05263 [condmat.stat-mech].

[15] V. B. Bulchandani, Phys. Rev. B 101, 041411 (2020), arXiv:1910.08266 [cond-mat.stat-mech].

[16] J. De Nardis, S. Gopalakrishnan, E. Ilievski, and R. Vasseur, arXiv e-prints (2020), arXiv:2003.13708 [cond-mat.stat-mech].

[17] B. Doyon, T. Yoshimura, and J.-S. Caux, Phys. Rev. Lett. 120, 045301 (2018), arXiv:1704.05482 [condmat.stat-mech].

[18] M. Fagotti, J. Phys. A 50, 034005 (2017), arXiv:1608.02869 [cond-mat.stat-mech].

[19] A. Urichuk, Y. Oez, A. Klümper, and J. Sirker, SciPost Phys. 6, 5 (2019), arXiv:1808.09033.

[20] D.-L. Vu and T. Yoshimura, SciPost Phys. 6, 23 (2019), arXiv:1809.03197 [cond-mat.stat-mech].

[21] Z. Bajnok and I. Vona, Phys. Lett. B 805, 135446 (2020), arXiv:1911.08525 [hep-th].

[22] H. Spohn, arXiv e-prints (2020), arXiv:2004.03802 [condmat.stat-mech].

[23] T. Yoshimura and H. Spohn, arXiv e-prints (2020), arXiv:2004.07113 [cond-mat.stat-mech].

[24] M. Borsi, B. Pozsgay, and L. Pristyák, Phys. Rev. X 10, 011054 (2020), arXiv:1908.07320 [cond-mat.stat-mech].

[25] B. Pozsgay, SciPost Phys. 8, 016 (2020), arXiv:1910.12833 [cond-mat.stat-mech].

[26] V. Korepin, N. Bogoliubov, and A. Izergin, Quantum inverse scattering method and correlation functions (Cambridge University Press, 1993).

[27] L. Faddeev, Acta Applicandae Mathematicae 39, 69 (1995).

[28] R. J. Baxter, Exactly solved models in statistical mechanics (London: Academic Press Inc, 1982).

[29] F. H. L. Essler, H. Frahm, F. Göhmann, A. Klümper, and V. E. Korepin, The One-Dimensional Hubbard Model (Cambridge University Press, Cambridge, 2005).

[30] B. Doyon and T. Yoshimura, SciPost Physics 2, 014 (2017), arXiv:1611.08225 [cond-mat.stat-mech].

[31] Supplemental Materials to "Algebraic construction of current operators in integrable spin chains".

[32] M. Lüscher, Nuclear Physics B 117, 475 (1976).

[33] E. Ilievski, M. Medenjak, and T. Prosen, Phys. 
Rev. Lett. 115, 120601 (2015), arXiv:1506.05049 [condmat.stat-mech].

[34] E. Ilievski, M. Medenjak, T. Prosen, and L. Zadnik, J. Stat. Mech. 6, 064008 (2016), arXiv:1603.00440 [condmat.stat-mech].

[35] G. Z. Fehér and B. Pozsgay, SciPost Phys. 8, 34 (2020), arXiv:1909.04470 [cond-mat.stat-mech].

[36] H. E. Boos and V. E. Korepin, J. Phys. A 34, 5311 (2001), hep-th/0104008.

[37] H. Boos, M. Jimbo, T. Miwa, F. Smirnov, and Y. Takeyama, Comm. Math. Phys. 286, 875 (2009), arXiv:0801.1176 [hep-th].

[38] M. Jimbo, T. Miwa, and F. Smirnov, J. Phys. A 42, 304018 (2009), arXiv:0811.0439 [math-ph].

[39] H. E. Boos, F. Göhmann, A. Klümper, and J. Suzuki, J. Phys. A 40, 10699 (2007), arXiv:0705.2716.

[40] J. Sato, B. Aufgebauer, H. Boos, F. Göhmann, A. Klümper, M. Takahashi, and C. Trippe, Phys. Rev. Lett. 106, 257201 (2011), arXiv:1105.4447 [cond-mat.str- el].

[41] B. Aufgebauer and A. Klümper, J. Phys. A 45, 345203 (2012), arXiv:1205.5702 [cond-mat.stat-mech].

[42] F. Göhmann and V. E. Korepin, J. Phys. A 33, 1199 (2000), arXiv:hep-th/9910253.

[43] J. M. Maillet and V. Terras, Nuclear Physics B 575, 627 (2000), hep-th/9911030.

[44] Note1.

[45] E. Sklyanin, J. Math. Sci. 80, 1861 (1996), arXiv:hepth/9212076.

[46] J. M. Maillet and G. Niccoli, J. Math. Phys. 59, 091417 (2018), arXiv:1807.11572 [math-ph].

[47] A. Cavaglià, N. Gromov, and F. Levkovich-Maslyuk, J. High Energy Phys. 2019, 52 (2019), arXiv:1907.03788 [hep-th].

[48] G. Niccoli, H. Pei, and V. Terras, arXiv e-prints (2020), arXiv:2005.01334 [math-ph]. 